\title{
Dichloroacetate induces protective autophagy in LoVo cells: involvement of cathepsin D/thioredoxin-like protein 1 and Akt-mTOR-mediated signaling
}

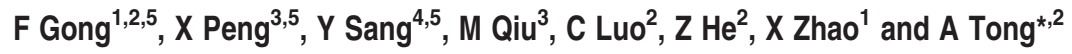

Dichloroacetate (DCA) is an inhibitor of pyruvate dehydrogenase kinase (PDK), and recently it has been shown as a promising nontoxic antineoplastic agent. In this study, we demonstrated that DCA could induce autophagy in LoVo cells, which were confirmed by the formation of autophagosomes, appearance of punctate patterns of LC3 immunoreactivity and activation of autophagy associated proteins. Moreover, autophagy inhibition by 3-methyladenine (3-MA) or Atg7 siRNA treatment can significantly enhance DCA-induced apoptosis. To determine the underlying mechanism of DCA-induced autophagy, target identification using drug affinity responsive target stability (DARTS) coupled with ESI-Q-TOF MS/MS analysis were utilized to profile differentially expressed proteins between control and DCA-treated LoVo cells. As a result, Cathepsin D (CTSD) and thioredoxin-like protein 1 (TXNL1) were identified with significant alterations compared with control. Further study indicated that DCA treatment significantly promoted abnormal reactive oxygen species (ROS) production. On the other hand, DCA-triggered autophagy could be attenuated by $\mathrm{N}$-acetyl cysteine (NAC), a ROS inhibitor. Finally, we demonstrated that the Akt-mTOR signaling pathway, a major negative regulator of autophagy, was suppressed by DCA treatment. To our knowledge, it was the first study to show that DCA induced protective autophagy in LoVo cells, and the potential mechanisms were involved in ROS imbalance and Akt-mTOR signaling pathway suppression.

Cell Death and Disease (2013) 4, e913; doi:10.1038/cddis.2013.438; published online 7 November 2013

Subject Category: Experimental Medicine

Autophagy is a cell survival mechanism during nutrient starvation that supplies vital components until conditions improve. ${ }^{1-3}$ Further, autophagy may facilitate the disposal of unfolded proteins and constitutes a mechanism utilized by tumor cells to survive hypoxic, metabolic and chemotherapeutic stress. ${ }^{4}$ It has been reported that inhibitors of autophagy was able to promote apoptosis induced by anticancer drugs, which suggested that autophagy could have a protective role in cancer cells. ${ }^{5-7}$ It has also been shown that cell death could be inhibited by suppressing the expression of some vital autophagy-associated genes, underscoring the important roles of autophagy in cell death. ${ }^{8,9}$ Thus, autophagy is a double-edged sword that can work both as a protector and a killer of cells. ${ }^{10,11}$

Dichloroacetate (DCA) is an inhibitor of pyruvate dehydrogenase kinase (PDK). It has been recently demonstrated as a promising nontoxic antineoplastic agent that promotes apoptosis of cancer cells. ${ }^{12-14}$ The anticancer effects of DCA were reported to be involved in cell cycle arrest and apoptosis induction. Until now, it is unknown whether autophagy can be induced by DCA and whether increased autophagy can affect the anticancer effects of DCA. Moreover, the underlying molecular mechanism of DCA-induced autophagy is largely unknown.

In the present study, we showed that DCA induced autophagy in human colon adenocarcinoma cell line, LoVo cells. Additionally, 3-methyladenine (3-MA)- or Atg7 siRNA-mediated inhibition of autophagy significantly enhanced DCA-induced apoptosis in LoVo cells. Through drug affinity responsive target stability (DARTS), MS and western blot analysis, we identified two redox-related proteins, Cathepsin D (CTSD) and thioredoxin-like protein 1 (TXNL1), which were significantly dysregulated following DCA treatment in LoVo cells. Further, abnormal reactive oxygen species (ROS) accumulation was observed in DCA-treated LoVo cells. Finally, we demonstrated that the Akt-mTOR signaling pathway, a major negative regulator of autophagy, was suppressed by DCA treatment. Therefore,

\footnotetext{
${ }^{1}$ Department of Gynecology and Obstetrics, Second West China Hospital; Cancer center, West China hospital, Sichuan University, Chengdu, China; ${ }^{2}$ State Key Laboratory of Biotherapy and Cancer Center, West China Hospital, West China Medical School, Sichuan University, Chengdu, China; ${ }^{3}$ Department of Medical Oncology, Cancer Center, State Key Laboratory of Biotherapy, West China Hospital, and Sichuan University, Chengdu, China and ${ }^{4}$ State Key Laboratory of Biotherapy, West China Hospital, and College of Life Science, Sichuan University, Chengdu, China

${ }^{*}$ Corresponding author: A Tong, State Key Laboratory of Biotherapy and Cancer Center, West China Hospital, West China Medical School, Sichuan University, Chengdu, China. Tel: +86 288516 4059; Fax: +86 288516 4060; E-mail: aipingtong @ gmail.com

${ }^{5}$ These authors contributed equally to this work.

Keywords: dichloroacetate; autophagy; DARTS; ROS; LoVo cells

Abbreviations: DCA, dichloroacetate; PDK, pyruvate dehydrogenase kinase; ESI-Q-TOF, electrospray ionization quadrupole time-of-flight; DARTS, drug affinity responsive target stability; CTSD, cathepsin D; ROS, reactive oxygen species; NAC, N-acetyl cysteine; 3-MA, 3-methyladenine; TEM, transmission electron microscopy; CAT, catalase; SOD, superoxide dismutase; TXNL1, thioredoxin-like protein 1

Received 13.6.13; revised 02.10.13; accepted 04.10.13; Edited by GM Fimia
} 
our current study provides new insight into DCA-induced autophagy.

\section{Results}

DCA induced autophagosome formation in LoVo cells. In the present study, five colorectal cancer cell lines (LoVo, SW620, HCT116, SW480 and LS174t) were used to investigate the cytotoxicity of DCA using an MTT assay. The results showed that LoVo cells were more sensitive to DCA treatment than other colorectal cancer cell lines, and thus were chosen for further study (Supplementary Table S1).

The ultrastructure of LoVo cells treated with $29 \mathrm{mM} \mathrm{DCA}$ or PBS for $48 \mathrm{~h}$ was analyzed using TEM. Numerous membrane bound vacuoles characteristic of autophagosomes were observed in the cytoplasm of DCA-treated cells, whereas membrane bound vacuoles could rarely be found when the cells were treated with PBS (Figure 1a).

To evaluate the recruitment of LC3-II to autophagosomes in response to DCA treatment, we examined localization of LC3 using a fluorescent Immunohistochemistry assay. As shown, punctate patterns of LC3 were observed in LoVo cells in response to $29 \mathrm{mM}$ DCA treatment for $24 \mathrm{~h}$, whereas it could not be seen in PBS-treated control cells. Notably, a significant increase in both the percentage of the cells with punctate patterns of LC3 and the LC3 dots per cell was observed in LoVo cells when the treatment time was prolonged to $48 \mathrm{~h}$ (Figure 1b). It can be observed that mitochondrion was entrapped into autophagosomal vacuoles in DCA-treated LoVo cells (Supplementary Figure S1), whereas there were not obvious changes in endoplasmic reticulum. Thus, DCA might induce selective mitochondrial autophagy.

LC3-I protein $(18 \mathrm{kDa})$ can be cleaved by autophagic stimuli to LC3-II (16 kDa), which is localized in autophagosome. As shown in Figure 1c, LC3-II protein was detectable in LoVo cells following $24 \mathrm{~h}$ of DCA treatment, whereas the expression of LC3-II was undetectable in PBS-treated control cells. These observations suggested that DCA induced autophagy in LoVo cells.

\section{Autophagic protein levels were augmented by DCA treatment in LoVo cells. To examine whether the key autophagic proteins were synergistically altered correspon- ding to DCA-induced autophagic process, western blot analysis was performed to examine the protein levels of Beclin 1, Atg7 and Atg12-Atg5 conjugate. As a result, the protein levels of Beclin 1, Atg7 and Atg12-Atg5 conjugate were markedly augmented following DCA treatment for $48 \mathrm{~h}$ when compared with PBS-treated control (Figure 1d).}

\footnotetext{
Autophagy inhibition promoted DCA-induced proliferation inhibition in LoVo cells. To determine the functional role of autophagy in DCA-induced proliferation inhibition, 3-MA, a widely used autophagy inhibitor (specific inhibitor of $\mathrm{PI}$ K), was used to block autophagic process in LoVo cells. ${ }^{15}$ LoVo cells were treated with 3-MA (5 mM), DCA (29 mM) or a combination for $48 \mathrm{~h}$. Apoptosis was assessed using an FCM assay. As shown in Figures $2 \mathrm{~A}-\mathrm{C}$, numerous membrane bound vacuoles characteristic of autophagosomes were
}

observed in the cytoplasm of DCA-treated cells, whereas membrane bound vacuoles could rarely be found when the cells were treated with DCA combined with 3-MA. DCAtriggered apoptosis was markedly enhanced in the presence of 3-MA, compared with treatment with DCA alone, 3-MA alone and PBS control (Figure 2D). Cell number and viability of DCA-treated LoVo cells were also significantly reduced in the presence of 3-MA compared with treatment with DCA alone, 3-MA alone and PBS control(Figures 2E and F). In order to further confirm DCA-induced autophagy had a protective role in cell apoptosis, we also used Atg7 siRNA to inhibit autophagy. Atg7 silencing by siRNA can inhibit the generation of autophagosomes, and thus prevent the induction of autophagy. ${ }^{16}$ As shown in Figures $2 \mathrm{G}$ and $\mathrm{H}$, cell number and viability were significantly reduced in Atg7 siRNA plus DCA treatment group compared with DCA alone, 3-MA alone and PBS control. Our data suggested that autophagy may have a protective role in LoVo cells following treatment with DCA.

DARTS analysis. As a small chemical molecule, it is very reasonable to think that there are other potential direct DCAbinding targets beside PDK. Here, DARTS methods were used to identify the potential molecular targets of DCA. As shown, DARTS analysis revealed two clearly visible differential bands between DCA-treated cells and the control group (Figure 3a). According to the theory of DARTS method, only the bands enriched in the DCA-treated group are thought to be DCA-protected proteins, that is to say, the potential DCA target proteins. However, the DARTS theory is not as mature as other classical methods, such as pulldown. Moreover, only two obvious bands were found to be differentially present in the DARTS assay. Hence, the two protein bands were all subjected to MS analysis and were identified to be CTSD and TXNL1, respectively (Figure 3a and Supplementary Table S2). We also examined the expression of the two proteins in LoVo cells after treatment with DCA by western blot. As shown in Figure 3b, DCA treatment upregulated the expression of CTSD while reduced the expression of TXNL1. However, whether the activities of CTSD and TXNL1 are inhibited or activated by DCA were not further analyzed in the present study. Moreover, these data do not exclude the possibility of other potential targets of lower abundance that were not evident by naked eye on the stained gels.

TXNL1 is able to undergo a rapid oxidation-reduction reaction and has a key role in maintaining the intracellular redox environment. ${ }^{17}$ CTSD is an aspartic protease, and its silencing was reported to be able to promote the expression of TXNL1 $1{ }^{18}$ It is known that ROS production has important roles in autophagy regulation. ${ }^{19}$ Hence, in the present study, we also examined the alteration in ROS level following DCA treatment and its potential roles in DCA-induced cell autophagy.

ROS accumulation mediated DCA-induced autophagy in LoVo cells. Intracellular ROS production was measured in LoVo cells at $48 \mathrm{~h}$ in response to treatment with different concentration of DCA. As shown, there was a marked dosedependent increase in ROS production compared with 
a
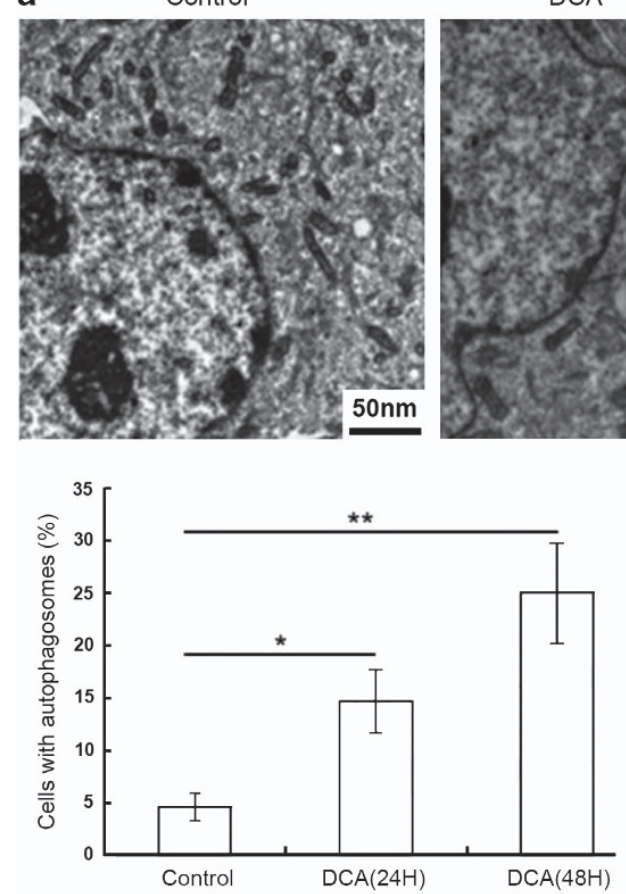

b
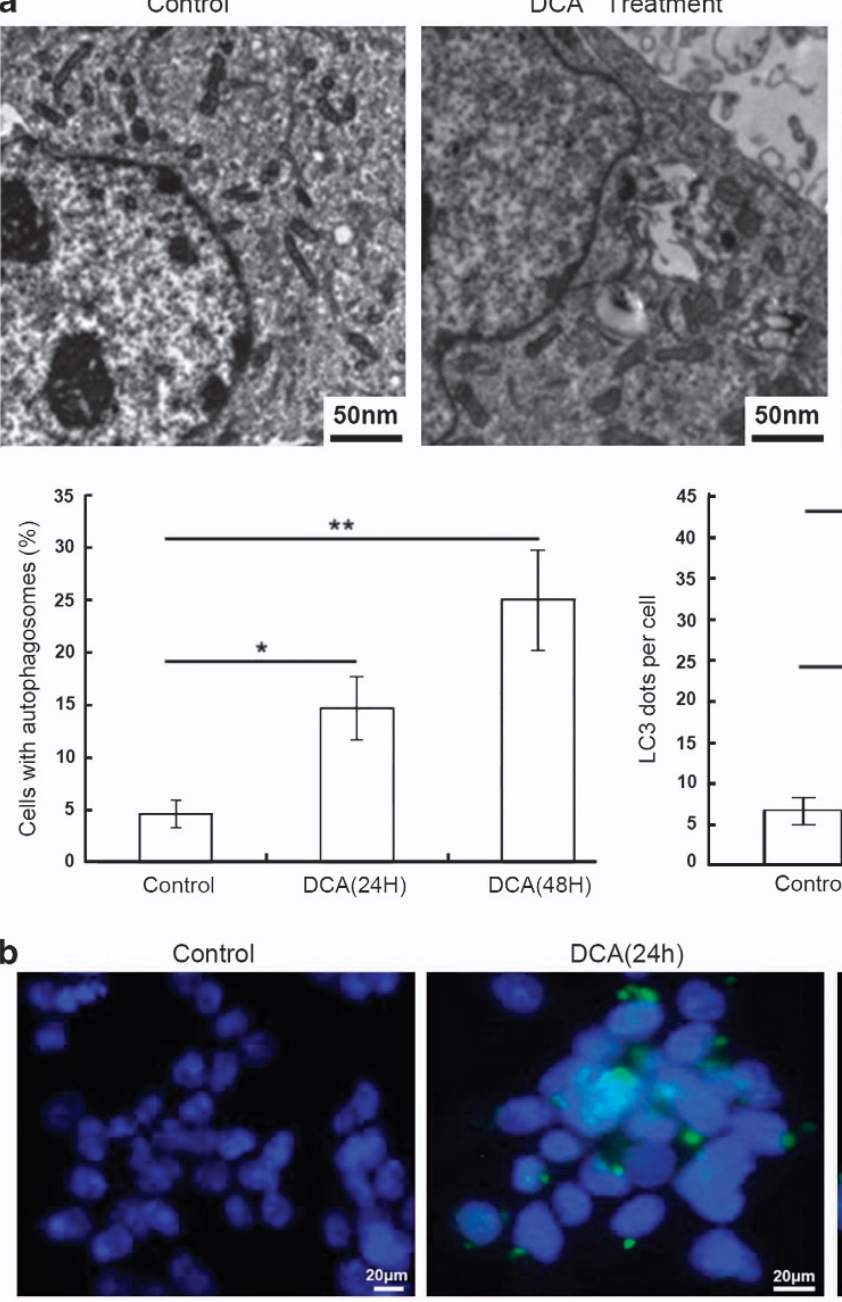

DCA Treatment (enlarged)
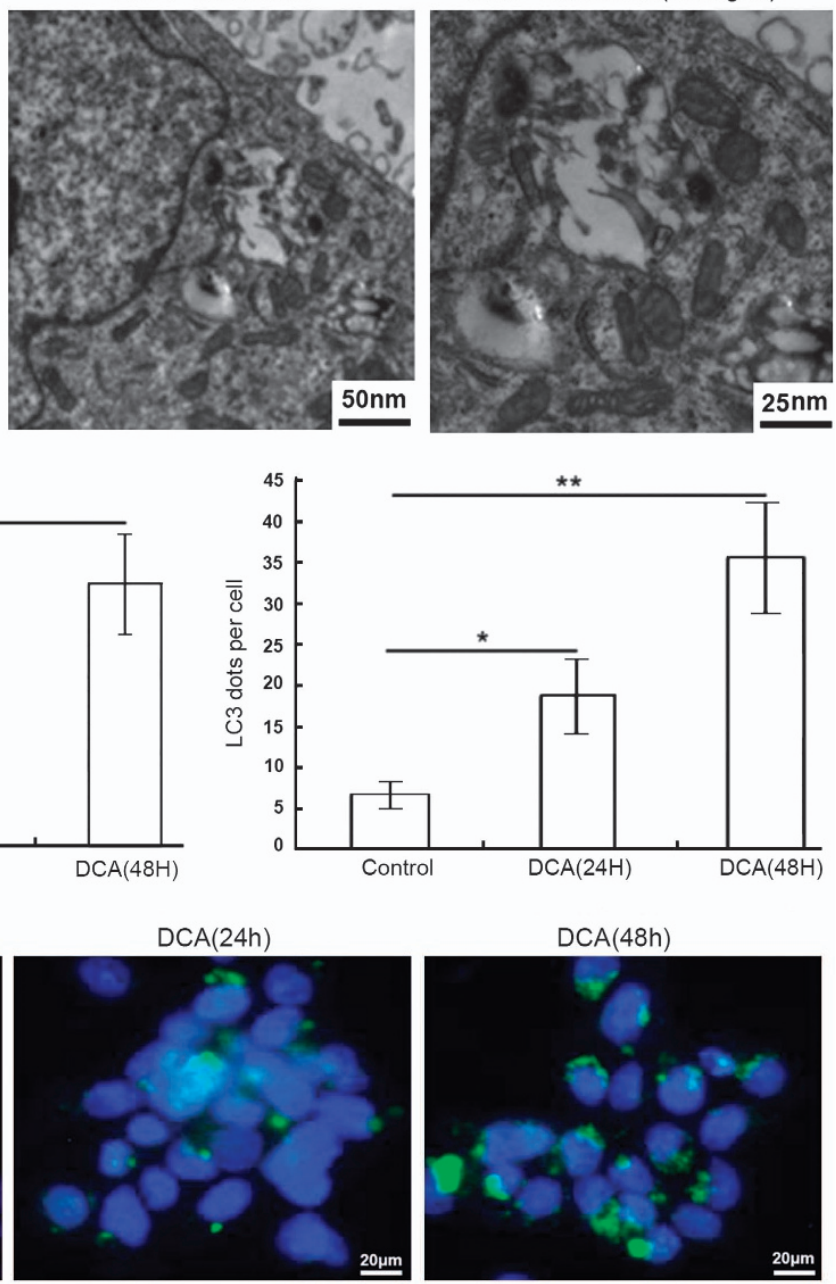

c

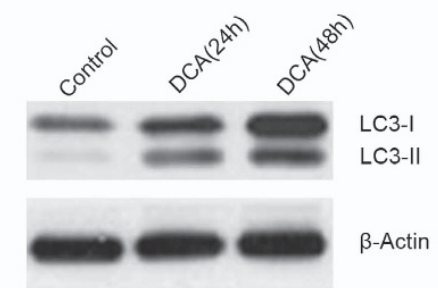

d
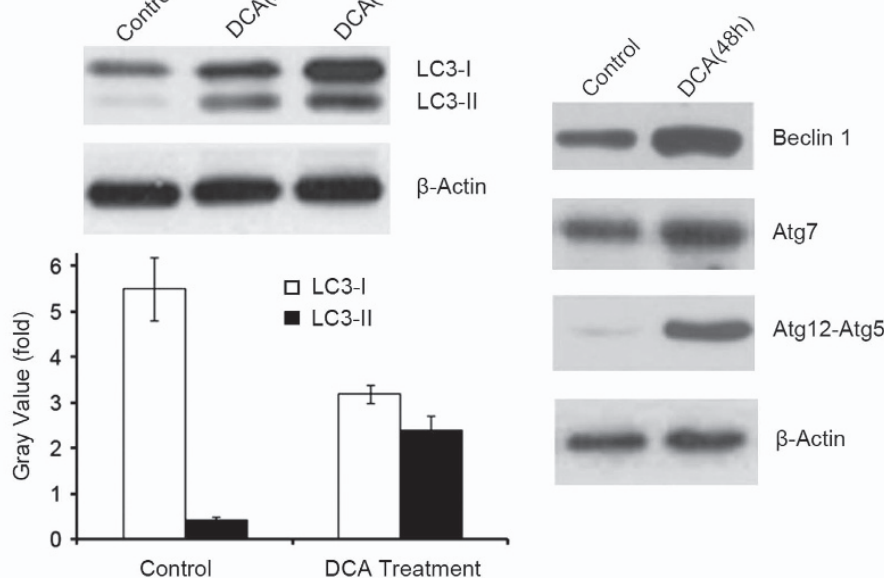

Figure 1 DCA induced autophagosome formation in LoVo cells. (a) Representative TEM depicting ultrastructures of LoVo cells treated with $29 \mathrm{mM}$ DCA for $48 \mathrm{~h}$. The percentage of the cells with autophagosomes was analyzed from at least 100 randomly chosen TEM fields. (b) The punctate patterns of LC3 staining was observed in the cytoplasm of the LoVo cells in response to $29 \mathrm{mM}$ DCA treatment for $24 \mathrm{~h}$, whereas LC3-GFP could not be seen in the control. Notably, a significant increase in both the percentage of the cells with punctate patterns of LC3 and the average number of punctate patterns of LC3 per cell was observed in LoVo cells when the treatment time was prolonged to $48 \mathrm{~h}$. (c) LC3-II protein was detectable in LoVo cells following DCA treatment for $24 \mathrm{~h}$, whereas the expression of LC3-II was undetectable in the control. (d) Western blot assay of Beclin 1, Atg7 and Atg12-Atg5 conjugate using lysates from LoVo cells treated with PBS (control) or $29 \mathrm{mM}$ DCA for $48 \mathrm{~h}$. $\beta$-Actin was used as a loading control. All data are representative of three independent experiments. ${ }^{*} P<0.05 ;{ }^{* *} P<0.01$ 
A
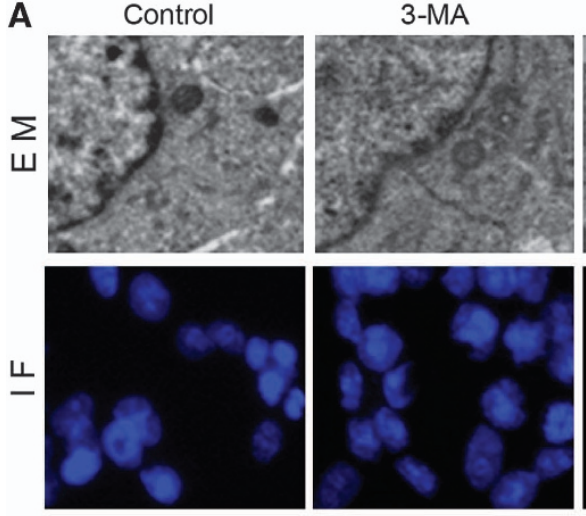

B

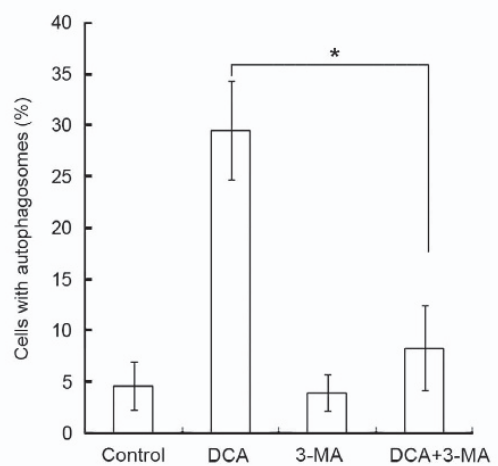

F

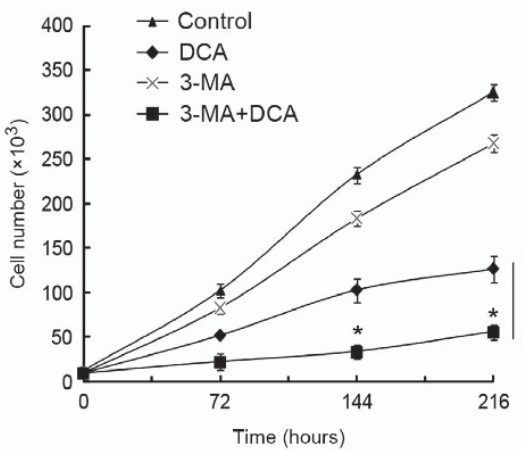

DCA


C ${ }_{50}$

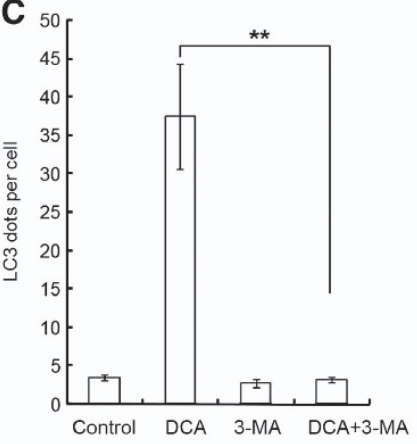

G

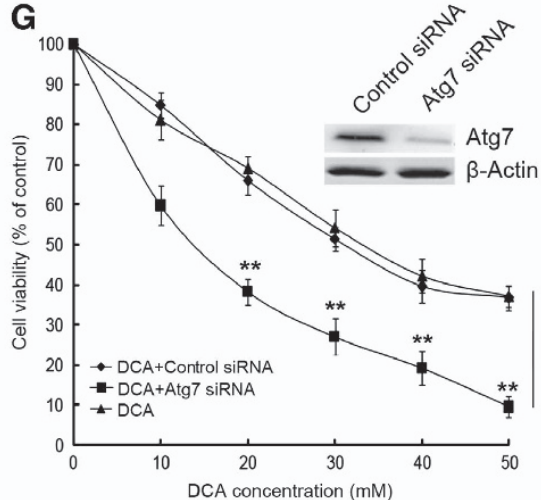




FL3 LOG

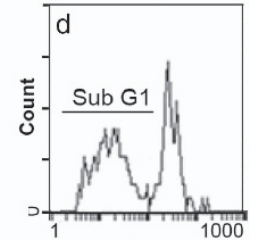

FL3 LOG

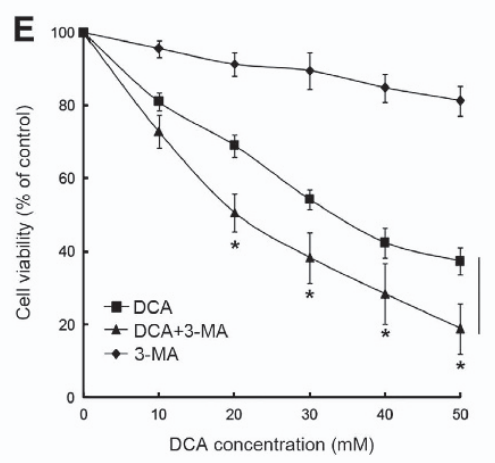

H

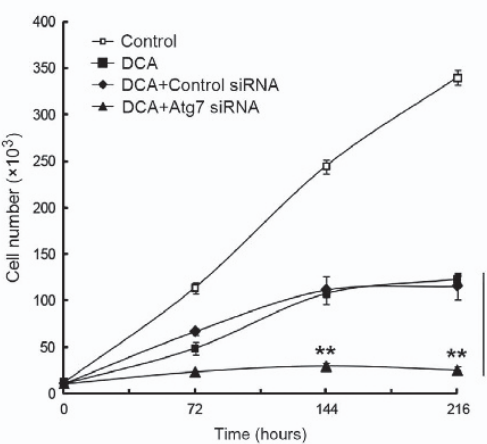

Figure 2 Inhibition of autophagy augmented DCA-induced proliferation inhibition and apoptosis in LoVo cells. (A) Representative images of transmission electron microscopy (upper) and LC3 immunofluorescence staining (lower). (B) The percentage of the cells with autophagosomes was markedly decreased when treated with 3-MA plus DCA compared with DCA alone $(P<0.05)$. (C) The LC3 dots per cell were significantly decreased in 3-MA plus DCA-treated LoVo cells compared with treatment with DCA alone $(P<0.01)$. (D) DCA-triggered apoptosis was markedly enhanced in the presence of 3-MA compared with treatment with DCA alone, 3-MA alone and PBS control $(P<0.01)$. a, PBS control; b, DCA; C, 3-MA; d, DCA plus 3-MA. $(E, F)$ Cell viability and cell number were also significantly reduced when treated with DCA plus 3-MA compared with DCA alone and 3-MA alone $(P<0.05)$. (G, H) Cell number and viability of DCA-treated LoVo cells were also significantly reduced when silencing Atg7 by siRNA $(P<0.01)$. Western blot was conducted to evaluate interference efficiency. ${ }^{*} P<0.05 ;{ }^{* *} P<0.01$

PBS-treated control (Figure 4a). We also measured the activity of superoxide dismutase (SOD) and catalase (CAT) in LoVo cells. As a result, the activity levels of SOD and CAT did not change significantly following treatment with different concentrations of DCA compared with the control group $(P>0.05)$ (Figures $4 \mathrm{~b}$ and $\mathrm{c}$ ).

Further, we analyzed the effects of blocking ROS production by $\mathrm{N}$-acetyl cysteine (NAC) on DCA-induced autophagy in LoVo cells. ${ }^{20,21}$ As shown, $10 \mathrm{mM}$ NAC treatment could completely reverse DCA-induced ROS production and significantly attenuated DCA-induced autophagy in LoVo cells (Figures $4 \mathrm{~d}-\mathrm{g}$ ). Moreover, western blot analysis indicated that the accumulation of LC3-II and the protein level of Beclin 1 was reduced while p62 was upregulated by DCA plus NAC treatment compared with DCA treatment alone (Figure $4 \mathrm{~h}$ ).

DCA inhibited the Akt-mTOR signaling pathway in LoVo cells. It is known that the Akt-mTOR signaling pathway is a 
a

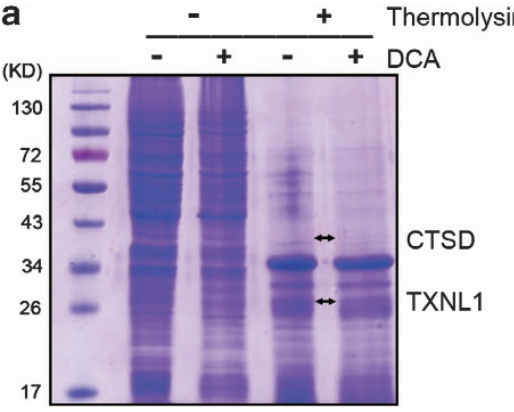

Figure 3 Identification of molecular targets of DCA using DARTS method. (a) After treatment with DCA (29 mM DCA), LoVo cell lysates were subjected to thermolysin digestion and Coomassie staining. On MS analysis, two differential protein bands were identified to be CTSD and TXNL1. (b) The protein levels of CTSD and TXNL1 were examined by western blotting in LoVo cells following DCA $(29 \mathrm{mM})$ treatment for $48 \mathrm{~h}$. $\beta$-Actin was used as a loading control. ${ }^{* *} P<0.01$



\section{Discussion}

DCA, a PDK inhibitor, has been studied as a promising drug in a variety of cancer models. ${ }^{12-14,22}$ PDK is located in the mitochondrial matrix and has important roles in cellular metabolism by regulating pyruvate dehydrogenase. Autophagy is an evolutionary conserved and lysosome-mediated examined whether DCA-induced autophagy is involved in the regulation of Akt-mTOR pathway. The phosphorylation status of both Akt and mTOR were examined by western DCA treatment for $48 \mathrm{~h}$ significantly inhibited the phosphorylation of both Akt (S473) and mTOR (S2448) compared with the control group. a

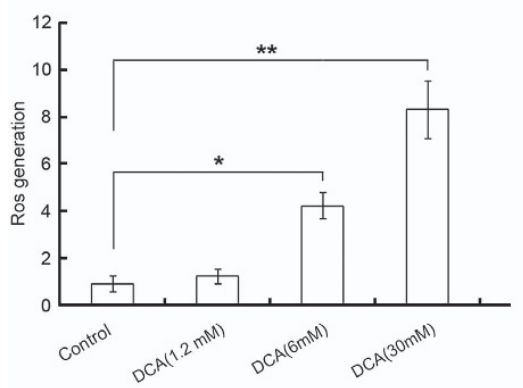

d

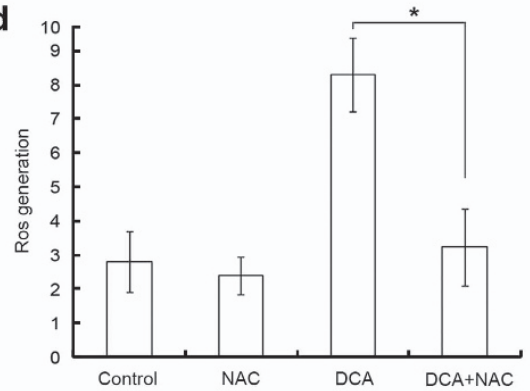

g


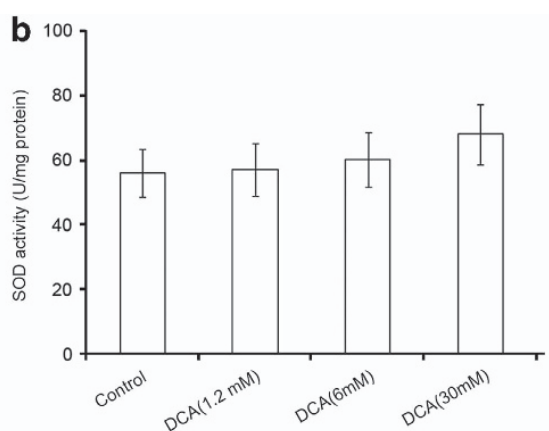

\section{e}
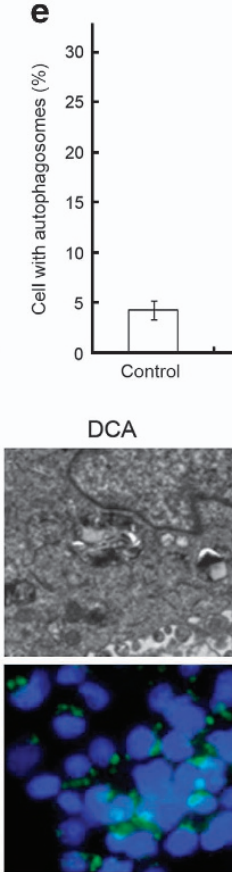

major negative regulator of autophagy. Hence, we further blot assay in DCA-treated LoVo cells. As shown in Figure 5,

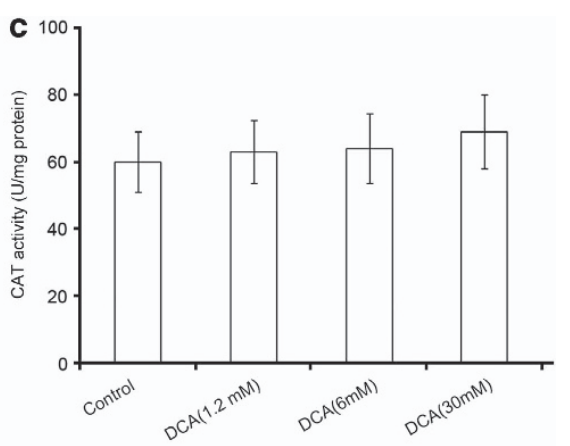

f



Figure 4 ROS mediated DCA-induced autophagy in LoVo cells. (a) Cellular ROS generation was assayed by DCFH-DA staining after treated with PBS, $1.2 \mathrm{mM}$ DCA, $6 \mathrm{mM} \mathrm{DCA}$ and $30 \mathrm{mM} \mathrm{DCA}$ for $48 \mathrm{~h}$ in LoVo cells. (b, c) The levels of both SOD and CAT activity did not change significantly when treated with different concentrations of DCA for $48 \mathrm{~h}(P>0.05)$. (d) Cellular ROS generation was reduced by treatment with a combination of $30 \mathrm{mM}$ DCA and $10 \mathrm{mM}$ NAC for $48 \mathrm{~h}$ compared with DCA treatment alone $(P<0.05)$. (e, f) The percentage of the cells with autophagosomes, and the LC3 dots per cell were markedly reduced in DCA plus NAC-treated LoVo cells compared with DCA alone $(P<0.05)$. (g) Representative picture of transmission electron microscopy (upper) and the punctate patterns of LC3 staining (lower). Cells were treated with DCA (30 mM) or NAC (10 mM) for $48 \mathrm{~h}$. (h) Western blots assay of autophagy-related proteins. $\beta$-Actin was used as a loading control. The data are representative of three independent experiments. ${ }^{\star} P<0.05 ;{ }^{* \star} P<0.01$ 




Figure 5 DCA inhibited the Akt-mTOR signaling pathway in LoVo cells. Cell lysates from LoVo cells either treated with PBS (control) or $29 \mathrm{mM} \mathrm{DCA} \mathrm{for} 48 \mathrm{~h}$ were subjected to western blot analysis. $\beta$-Actin was used as a loading control

process, which involves delivery of cytoplasmic material to lysosome. ${ }^{1-3,23}$ Accumulating studies have documented that protective autophagy could antagonize apoptotic cell death induced by cancer chemotherapy and radiotherapy. ${ }^{5-7,24}$ Until now, it is unknown whether autophagy can be induced by DCA.

In this study, we showed that DCA could induce autophagy in LoVo cells, as evidenced by appearance of autophagic vacuoles, punctate patterns of LC3 immunoreactivity and accumulation of Beclin 1, Atg12-Atg5 and Atg7, which are essential for autophagosome formation. Moreover, inhibition of autophagy by 3-MA or Atg7 siRNA significantly enhanced DCA-induced apoptosis in LoVo cells, suggesting autophagy could have a protective role in tumor cells treated with DCA. Hence, it is possible that lower concentration of DCA may show antitumor effects when using combined with autophagy inhibitors.

On DARTS and western blot analysis, we found two redox balance-associated proteins, CTSD and TXNL1, were dysregulated by DCA treatment. TXNL1 is an important intracellular antioxidative protein that regulates the reduction of ROS. ${ }^{17,25}$ CTSD is a lysosomal aspartyl protease, and it was reported that CTSD silencing can increase the expression of TXNL1 and subsequently reduce ROS content in $\mathrm{H}_{2} \mathrm{O}_{2}$-treated endothelial cells. ${ }^{18}$ On performing ROS chemiluminescence assay, we also found that DCA treatment significantly augmented intracellular ROS accumulation. ROS are generally small, short-lived and highly reactive molecules, which can be produced through the interaction of ionizing radiation with biological molecules, as an unavoidable byproduct of cellular respiration or synthesized by dedicated enzymes. ${ }^{26}$ An increasing number of investigations have shown that ROS accumulation is a stimulus to trigger cell autophagy. ${ }^{19-21}$ In the present study, we found that both DCA-induced autophagosome and accumulation of LC3-II could be significantly attenuated by treatment with ROS inhibitor NAC, suggesting that ROS may act as a positive mediator of DCA-induced autophagy. However, according to the theory of DARTS method, only the bands enriched in DCA-treated group are thought to be potential DCA target proteins. Hence, what are the functional roles of CTSD and TXNL1 in the antitumor effects of DCA need further study. Moreover, whether the enzyme activity of CTSD is inhibited or activated by direct binding of DCA is an interesting question that deserves to be investigated. In contrast, what relationship exists among the dysregulation of CTSD and TXNL1 and the known target PDK needs further investigation.

The Akt-mTOR signaling pathway is an important negative regulator of autophagy. In this study, we showed that phosphorylation of both Akt and mTOR was inhibited by DCA treatment, suggesting that inhibition of Akt-mTOR signaling may contribute to DCA-induced autophagy in LoVo cells. PDK is located in the mitochondrial matrix. In Supplementary Figure S1, we showed that mitochondria were obviously damaged by DCA treatment, whereas ER was not. Hence, crosstalk among mitochondrial damage, ROS stress, autophagy and the Akt-mTOR signaling pathway should be studied.

In conclusion, we found DCA inhibited proliferation and induced apoptosis in LoVo cells. DCA treatment can induce significant cell autophagy. Autophagy has a selfprotection role in LoVo cells exposed to DCA because enhanced apoptosis was detected by blocking autophagy when treated with DCA. We found two redox balancerelated proteins, $C T S D$ and $T X N L 1$, were significantly dysregulated by DCA treatment. Moreover, the present study also showed that there was a marked ROS accumulation following DCA treatment, and DCA-induced cell autophagy can be inhibited by ROS inhibitor (NAC) treatment. Finally, we demonstrated that the Akt-mTOR signaling pathway, a major negative regulator of autophagy, was suppressed by DCA treatment. The present study provided new clues with respect to the underlying mechanisms of the antitumor effects of DCA.

\section{Materials and Methods}

Cell culture and reagents. Human colon adenocarcinoma LoVo cell line was purchased from ATCC (Manassas, VA, USA). Cells were cultured in RPMl1640 supplemented with $10 \%$ fetal bovine serum, $10^{5} \mathrm{U} / \mathrm{l}$ penicillin and $100 \mathrm{mg} / \mathrm{l}$ streptomycin (Sigma, St. Louis, MO, USA; P0781) at $37^{\circ} \mathrm{C}$ in an atmosphere containing $5 \% \mathrm{CO}_{2}$.

Reagents used in this study were DCA (Sigma-aldrich, St. Louis, MO, USA; 347795), 3-MA (Sigma-aldrich, M9281), NAC (Sigma, A9165), SOD activity assay kit (Biovision, Milpitas, CA, USA; k335-100), Catalase assay kit (Cayman Chemical, Ann Arbor, MI, USA; 707002), Lipofectamine 2000 reagent (Invitrogen, Carlsbad, CA, USA; 11668-019). Antibodies used in this study were Beclin 1 (Santa Cruz, Santa Cruz, CA, USA; sc-11427), LC3 (Abcam, Cambridge, England; ab58610), cathepsin D (EMD Biosciences, Darmstadt, Germany; IM03), Atg12-Atg5 (Abcam, ab78073), Atg7 (Abcam, ab53255), $\beta$-actin (Santa Cruz, sc-1616), TXNL1 (Santa Cruz, sc-58439), Akt (Cell Signaling, Boston, MA, USA; 4685), phospho-Akt (Cell Signaling, 4051), mTOR (Cell Signaling, 2983), p-mTOR (Cell Signaling, 2971), 
horseradish peroxidase (HRP)-conjugated anti-rabbit secondary antibody (Santa Cruz, sc-2004), HRP-conjugated anti-mouse secondary antibody (Santa Cruz, sc-2005).

Transmission electron microscopy. Cells were pelleted and fixed in paraformaldehyde, $0.1 \%$ glutaraldehyde in $0.1 \mathrm{M}$ sodium cacodylate for $2 \mathrm{~h}$, postfixed with $1 \% \mathrm{O}_{\mathrm{s}} \mathrm{O}_{4}$ for $1.5 \mathrm{~h}$, washed and finally stained for $1 \mathrm{~h}$ in $3 \%$ aqueous uranyl acetate. Samples were then rinsed with water again, dehydrated with graded alcohol (50, 75 and $95-100 \%$ alcohol) and embedded in Epon-Araldite resin (Canemco, 034). Ultrathin sections were cut on a Reichert Ultramicrotome, counterstained with $0.3 \%$ lead citrate and examined on a Philips EM420 transmission electron microscope. ${ }^{27}$

Cell viability, proliferation and apoptosis assays. For viability assay, cells were seeded at $5 \times 10^{3}$ cells per well in 96-well plates and were allowed to attach overnight at $37^{\circ} \mathrm{C}$. Afterwards, medium containing agents were added to each well, and cells were further cultured at $37^{\circ} \mathrm{C}$ for the indicated times. Cell viability was estimated using an MTT. The absorbance was measured at $570 \mathrm{~nm}$ with a microplate reader (Bio-Rad, Berkeley, CA, USA). Cell proliferation was analyzed by cell counting. Briefly, cells were trypsinized and replated in 24-well plates at a density of 10000 cells/well. Cells were incubated for $24 \mathrm{~h}$ to allow for attachment. Afterwards, medium containing agents were added to each well, and cells were counted at days $0,3,6$ and 9 with a cell counter. Cell apoptosis was analyzed by flow cytometry analysis using propidium iodide staining. All assays were independently performed at least three times.

LC3 immunofluorescence assay. Cells were washed with PBS and then fixed with paraformaldehyde (4\% w:v). After rinsing in PBS, the cells were blocked with $0.1 \%$ Triton $\mathrm{X}-100$ containing $1 \%$ bovine serum albumin in PBS for $1 \mathrm{~h}$. This was followed by incubation with an LC3 antibody for $16 \mathrm{~h}$ at $4{ }^{\circ} \mathrm{C}$ in a humidified chamber. After three washes in PBS, cells were incubated in a secondary antibody for $2 \mathrm{~h}$ at room temperature. Finally, cells were rinsed in PBS, coverslipped and examined with fluorescence microscopy.

Detection of mitochondrial autophagy. In order to study whether DCA induces mitochondrial autophagy, GFP-LC3 and DsRed-Mito plasmids were co-transfected in LoVo cells. Endoplasmic reticulum was stained with ER-Tracker Red (Beyotime, C1041).

siRNA preparation and transfection. Atg7 knockdowns were accomplished by transfecting LoVo cells with siRNA. Atg7 and control siRNA were synthesized by RiboBio (Guangzhou, China). The siRNA sequences against atg7 were previously described. ${ }^{28}$ Cells $\left(3 \times 10^{5}\right)$ were transfected with $1 \mu \mathrm{g}$ siRNA using $2.5 \mu \mathrm{g}$ Lipofectamine 2000 reagent (Invitrogen). Scrambled siRNA, which consists of a scrambled sequence that will not lead to specific degradation of any known cellular mRNA, was employed as negative control.

DARTS analysis. After treatment with $29 \mathrm{mM}$ DCA for $30 \mathrm{~min}$, LoVo cells were lysed with M-PER (Pierce, Rockford, IL, USA) supplemented with protease and phosphatase inhibitors. After centrifugation (14000 r.p.m. using Beckman Coulter Microfuge, $15 \mathrm{~min}$ ), lysates were diluted to the same final volume and protein concentration with M-PER and proteolysed in reaction buffer $(50 \mathrm{mM}$ Tris$\mathrm{HCl}, \mathrm{pH} 8.0,50 \mathrm{mM} \mathrm{NaCl}, 10 \mathrm{mM} \mathrm{CaCl}_{2}$ ). All steps were performed on ice or at $4{ }^{\circ} \mathrm{C}$ to help prevent premature protein degradation. Each sample was then quickly warmed to room temperature and proteolysed with $1 \mu \mathrm{g}$ thermolysin for every $15 \mu \mathrm{g}$ lysate for $10 \mathrm{~min}$. To stop proteolysis, $0.5 \mathrm{M}$ EDTA (pH 8.0) was added to each sample at a 1:10 ratio, mixed well. ${ }^{29-31}$

Mass spectrometry analysis. Gel bands were cut out and prepared for mass spectrometry analysis with trypsin digestion. Peptides were analyzed using a Q-TOF mass spectrometer (Micromass, Manchester, UK) fitted with an ESI source (Waters, Manchester, UK). ${ }^{32}$

Reactive oxygen species (ROS) measurement. Intracellular ROS level was detected by staining cells with $2^{\prime}, 7^{\prime}$ - dichloro-fluoresce in diacetate (DCFH-DA) (GENMED, GMS10016.2) according to the manufacturer's instructions. ${ }^{33}$ The DCFH-DA signal was measured at $530 \mathrm{~nm}$ with a fluorimeter.

Superoxide dismutase and catalase activity assay. SOD activity was analyzed using a RASOD Assay Kit (Biovision) and CAT activity was measured using a Catalase Assay Kit (Cayman Chemical).
Western blot. Proteins were extracted in RIPA buffer $(50 \mathrm{mM}$ Tris-base, $1.0 \mathrm{mM}$ EDTA, $150 \mathrm{mM} \mathrm{NaCl}, 0.1 \%$ SDS, $1 \%$ TritonX-100, $1 \%$ Sodium deoxycholate, $1 \mathrm{mM}$ PMSF) and quantified using the DC protein assay kit. Samples were separated by $12 \%$ SDS-PAGE and transferred to PVDF membranes. The membranes were blocked overnight with PBS containing $0.1 \%$ Tween 20 in $5 \%$ skimmed milk at $4{ }^{\circ} \mathrm{C}$, and subsequently probed by the primary antibodies rabbit-anti-Beclin 1 (diluted 1:500), rabbit-anti-Atg12-Atg5 (diluted $1: 500$ ), rabbit-anti-Atg7 (diluted $1: 400$ ), rabbit-anti-LC3 (diluted $1: 400$ ), mouseanti-TXNL1 (diluted 1:500), mouse-anti-cathepsin D (diluted 1:500), rabbit-antiAkt, mouse-anti-Phospho-Akt, rabbit-anti-mTOR and rabbit-anti-Phospho-mTOR used all in the dilution of $1: 1000$. Blots were incubated with the respective primary antibodies for $2 \mathrm{~h}$ at room temperature. After washing three times in Tris-buffered saline with Tween $20(20 \mathrm{mM}$ Tris- $\mathrm{HCl}, 150 \mathrm{mM} \mathrm{NaCl}, 0.1 \%$ Tween 20$)$, the blots were incubated with an HRP-conjugated anti-rabbit secondary antibody (diluted $1: 5000$ ) or an HRP-conjugated anti-mouse secondary antibody (diluted 1:5000) for $2 \mathrm{~h}$ at room temperature. Blots were visualized by western chemiluminescence reagents (Millipore, WBKLS0500). Densitometric quantitation of blotting strips was determined using the Quality-One software. $\beta$-Actin was used as an internal control.

Statistical data analysis. Comparisons between two groups were performed by Student's $t$ test. Statistical significance was defined as ${ }^{*} P<0.05$; ${ }^{* *} P<0.01$.

\section{Conflict of Interest}

The authors declare no conflict of interest.

Acknowledgements. This work was supported by the grants from the National Natural Sciences Foundation of China (31171370) and the Postdoctoral 52nd Foundation of China (2012M521701).

1. Kundu M, Thompson CB. Autophagy: basic principles and relevance to disease. Ann Rev Pathol 2008; 3: 427-455.

2. Levine B, Kroemer G. Autophagy in the pathogenesis of disease. Cell 2008; 132 : 27-42.

3. Mizushima N, Levine B, Cuervo AM, Klionsky DJ. Autophagy fights disease through cellular self-digestion. Nature 2008; 451: 1069-1075.

4. Choi AM, Ryter SW, Levine B. Autophagy in human health and disease. New Engl J Med 2013; 368: 1845-1846.

5. Wang K, Liu R, Li J, Mao J, Lei Y, Wu J et al. Quercetin induces protective autophagy in gastric cancer cells: involvement of Akt-mTOR- and hypoxia-induced factor 1alpha-mediated signaling. Autophagy 2011; 7: 966-978.

6. Cheng P, Ni Z, Dai X, Wang B, Ding W, Rae Smith A et al. The novel BH-3 mimetic apogossypolone induces Beclin-1- and ROS-mediated autophagy in human hepatocellular carcinoma cells. Cell Death Disease 2013; 4: e489.

7. Lin JF, Tsai TF, Liao PC, Lin YH, Lin YC, Chen HE et al. Benzyl isothiocyanate induces protective autophagy in human prostate cancer cells via inhibition of mTOR signaling. Carcinogenesis 2013; 34: 406-414.

8. Pyo JO, Jang MH, Kwon YK, Lee HJ, Jun Jl, Woo HN et al. Essential roles of Atg5 and FADD in autophagic cell death: dissection of autophagic cell death into vacuole formation and cell death. J Biol Chem 2005; 280: 20722-20729.

9. Pattingre S, Tassa A, Qu X, Garuti R, Liang XH, Mizushima N et al. Bcl-2 antiapoptotic proteins inhibit Beclin 1-dependent autophagy. Cell 2005; 122: 927-939.

10. Kimura T, Takabatake $Y$, Takahashi A, Isaka Y. Chloroquine in cancer therapy: a doubleedged sword of autophagy. Cancer Res 2013; 73: 3-7.

11. Parkhitko AA, Favorova OO, Henske EP. Autophagy: mechanisms, regulation, and its role in tumorigenesis. Biochemistry Biokhimiia 2013; 78: 355-367.

12. Michelakis ED, Webster L, Mackey JR. Dichloroacetate (DCA) as a potential metabolictargeting therapy for cancer. Br J Cancer 2008; 99: 989-994.

13. Papandreou I, Goliasova T, Denko NC. Anticancer drugs that target metabolism: Is dichloroacetate the new paradigm? Int J Cancer 2011; 128: 1001-1008.

14. Lingohr MK, Bull RJ, Kato-Weinstein J, Thrall BD. Dichloroacetate stimulates glycogen accumulation in primary hepatocytes through an insulin-independent mechanism. Toxicol Sci 2002; 68: 508-515.

15. Ito S, Koshikawa N, Mochizuki S, Takenaga K. 3-Methyladenine suppresses cell migration and invasion of HT1080 fibrosarcoma cells through inhibiting phosphoinositide 3-kinases independently of autophagy inhibition. Int J Oncol 2007; 31: 261-268.

16. Komatsu M, Waguri S, Ueno T, Iwata J, Murata S, Tanida I et al. Impairment of starvationinduced and constitutive autophagy in Atg7-deficient mice. J Cell Biol 2005; 169: 425-434. 
17. Jacquot JP, de Lamotte F, Fontecave M, Schurmann $P$, Decottignies $P$, Miginiac-Maslow $M$ et al. Human thioredoxin reactivity-structure/function relationship. Biochem Biophys Res Commun 1990; 173: 1375-1381.

18. Haendeler J, Popp R, Goy C, Tischler V, Zeiher AM, Dimmeler S et al. and $\mathrm{H}_{2} \mathrm{O}_{2}$ stimulate degradation of thioredoxin-1: implication for endothelial cell apoptosis. J Biol Chem 2005; 280: 42945-42951.

19. Scherz-Shouval R, Elazar Z. ROS mitochondria and the regulation of autophagy. Trends Cell Biol 2007; 17: 422-427.

20. Chen Y, McMillan-Ward E, Kong J, Israels SJ, Gibson SB. Oxidative stress induces autophagic cell death independent of apoptosis in transformed and cancer cells. Cell Death Differ 2008; 15: 171-182.

21. Haga S, Terui K, Zhang HQ, Enosawa S, Ogawa W, Inoue $\mathrm{H}$ et al. Stat3 protects against Fas-induced liver injury by redox-dependent and -independent mechanisms. J Clin Invest 2003; 112: 989-998.

22. Tong J, Xie G, He J, Li J, Pan F, Liang H. Synergistic antitumor effect of dichloroacetate in combination with 5-fluorouracil in colorectal cancer. J Biomed Biotechnol 2011; 2011: 740564.

23. Levine B. Cell biology: autophagy and cancer. Nature 2007; 446: 745-747.

24. Yousefi S, Perozzo R, Schmid I, Ziemiecki A, Schaffner T, Scapozza L et al. Calpainmediated cleavage of Atg5 switches autophagy to apoptosis. Nature Cell Biol 2006; 8: 1124-1132.

25. Dayer R, Fischer BB, Eggen RI, Lemaire SD. The peroxiredoxin and glutathione peroxidase families in Chlamydomonas reinhardtii. Genetics 2008; 179: 41-57.

26. Chen Y, McMillan-Ward E, Kong J, Israels SJ, Gibson SB. Mitochondrial electron-transport-chain inhibitors of complexes I and II induce autophagic cell death mediated by reactive oxygen species. J Cell Sci 2007; 120: 4155-4166.
27. Paglin S, Lee NY, Nakar C, Fitzgerald M, Plotkin J, Deuel B et al. Rapamycin-sensitive pathway regulates mitochondrial membrane potential, autophagy, and survival in irradiated MCF-7 cells. Cancer Res 2005; 65: 11061-11070.

28. Lavieu G, Scarlatti F, Sala G, Carpentier S, Levade T, Ghidoni R et al. Regulation of autophagy by sphingosine kinase 1 and its role in cell survival during nutrient starvation. J Biol Chem 2006; 281: 8518-8527.

29. Lomenick B, Hao R, Jonai N, Chin RM, Aghajan M, Warburton S et al. Target identification using drug affinity responsive target stability (DARTS). Proc Natl Acad Sci USA 2009; 106 21984-21989.

30. Lomenick B, Olsen RW, Huang J. Identification of direct protein targets of small molecules. ACS Chem Biol 2011; 6: 34-46.

31. Ambrosino C, Tarallo R, Bamundo A, Cuomo D, Franci G, Nassa G et al. Identification of a hormone-regulated dynamic nuclear actin network associated with estrogen receptor alpha in human breast cancer cell nuclei. Mol Cell Proteomics 2010; 9 : 1352-1367.

32. Peng XC, Gong FM, Zhao YW, Zhou LX, Xie YW, Liao HL et al. Comparative proteomic approach identifies PKM2 and cofilin-1 as potential diagnostic, prognostic and therapeutic targets for pulmonary adenocarcinoma. PLoS One 2011; 6: e27309.

33. McMurtry MS, Archer SL, Altieri DC, Bonnet S, Haromy A, Harry G et al. Gene therapy targeting survivin selectively induces pulmonary vascular apoptosis and reverses pulmonary arterial hypertension. J Clin Invest 2005; 115: 1479-1491.

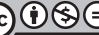

Cell Death and Disease is an open-access journal published by Nature Publishing Group. This work is licensed under a Creative Commons Attribution-NonCommercialNoDerivs 3.0 Unported License. To view a copy of this license, visit http://creativecommons.org/licenses/by-nc-nd/3.0/

Supplementary Information accompanies this paper on Cell Death and Disease website (http://www.nature.com/cddis) 